[Student Paper ]

\title{
MOS Ge/Si Quantum Dot Infrared Photodetectors with Quantum Dot and Wetting Layer Responses
}

\author{
B.-C. Hsu", S. T. Chang ", P.-S. Kuo", P. S. Chen ${ }^{c}$, C. W. Liü, , J.-H. Lu", and C. H. Kuan" \\ ${ }^{a}$ Department of Electrical Engineering and Graduate Institute of Electronics Engineering, \\ National Taiwan University, Taipei, Taiwan, R. O. C. \\ ${ }^{\mathrm{h}}$ Department of Electronic Engineering, Chung Yuan Christian University, \\ Chung-Li, Taiwan, R. O.C. \\ ${ }^{c}$ ERSO/ITRI, Hsinchu, Taiwan, R. O.C. E-mail: chee@occ.ee.ntu.edu.tw
}

Due to low dark current, high operation temperature and normal incident detection, the quantum dot infrared photodetector (QDIP) using the intervalence band transitions is very attractive in the military, medical, astronomical and other applications. The simple metal-insulator-semiconductor (MIS) structure with tunneling insulator can make the Ge/Si QDIP compatible with Si ULSI process, and make it possible to have convenient and accurate body temperature measurement using a Si chip. A 5-period self-assembled $\mathrm{Ge} / \mathrm{Si}$ quantum dots with 3 $\mathrm{nm}$ wetting layers (quantum well) grown by UHV/CVD (Fig. I) is fabricated into MIS tunneling diodes [1] with low temperature $\left(50^{\circ} \mathrm{C}\right)$ liquid phase deposition (LPD) oxide and oxynitride [2]. The existence of $N$ in oxynitride is confirmed by SIMS (Fig. 2).

The dark current of MIS tunneling diode is dominated by thermal generation of electron-hole pairs through the defects in the depletion region and at the $\mathrm{Si}_{/} / \mathrm{SiO}_{2}$ interface (Fig. 3). The LPD oxynitride has a low interface density, and thus has a low dark current as compared to LPD oxide (Fig. 4). The thermally-generated electrons tunnel through the insulator layer and the thermally-generated holes in deep depletion region as well as the holes tunneling from gate electrode are swept toward the substrate. Due to the large valence band offset formed in $\mathrm{Ge} / \mathrm{Si}$ heterojunctions, the hole densities are high in the Ge quantum layers. The Ge quantum dots are placed in the depletion region at inversion bias to have electrical field to drift the photo-excited holes. Under infrared exposures, the confined holes have bound-to-bound (quantum well) and bound-to-continuum (quantum det)-transitions (Fig. 3).

The spectral responsivity of the NMOS Ge/Si QDIP with oxynitride has two absorption regions (2 3 $\mu \mathrm{m}$ and $3 \sim 10 \mu \mathrm{m}$, Fig. 5). For $3 \sim 10 \mu \mathrm{m}$ detection, the peak wavelength is located at $6.8 \mu \mathrm{m}$ and the maximum operating temperature is about $140 \mathrm{~K}$. For $2 \sim 3 \mu \mathrm{m}$ detection, the peak wavelength is located at $2.7 \mu \mathrm{m}$ and the operating temperature is up to $200 \mathrm{~K}$ (Fig. 6). Since the operating temperature is higher for $2 \sim 3 \mu \mathrm{m}$ detection, this short wavelength response mainly comes from the quantum dot structure due to the better quantum confinement, while the $3 \sim 10 \mu \mathrm{m}$ response is dominated by the wetting layer structure. The photoluminescence (PL) spectrum aiso confirms that the short wavelength response comes form the quantum dot structure with the larger barrier for bound-to-continuum transition $\left(0.3 \sim 0.4 \mathrm{eV}\right.$, Fig. 7) [3]. The peak detectivity is $10^{10}$ and $10^{9} \mathrm{~cm} \cdot \mathrm{Hz}^{1 / 2} / \mathrm{W}$ for $6.8 \mu \mathrm{m}$ and $2.7 \mu \mathrm{m}$, respectively (Fig. 8). The MOS Ge/Si QDIP with LPD oxide has a stronger responsivity as compared to the device with LPD oxynitride (Fig. 9). However, the operating temperature is only $80 \mathrm{~K}$ for $3 \sim 10 \mu \mathrm{m}$ detection and is $120 \mathrm{~K}$ for $2 \sim 3 \mu \mathrm{m}$ detection (Fig. 10). The lower operation temperature is due to the larger dark current of oxide devices (Fig. 4). The $7.5 \mu \mathrm{m}$ detectivity at $60 \mathrm{~K}$ reaches $2 \times 10^{11} \mathrm{~cm} \cdot \mathrm{Hz}^{1 / 2} / \mathrm{W}$ (Fig. 11 ).

The valance band bound state energy of wetting layer is calculated by $k \cdot p$ method. By calculating the total intersubband transitions, a absorption peak is located at $7.5 \mu \mathrm{m}$ (Fig. 12). The theoretical results show good agreement with the experimental spectrum and confirmed that the long wavelength response mainly comes from the wetting layer structure (quantum well).

The MOS Ge/Si QDIPs for $2-10 \mu \mathrm{m}$ are successfully demonstrated. Using oxynitride as gate dielectric, the operating temperature reaches $140 \mathrm{~K}$ for $3 \sim 10 \mu \mathrm{m}$ and is up to $200 \mathrm{~K}$ for $2 \sim 3 \mu \mathrm{m}$ detection. The quantum dot structure is responsible for $2-3 \mu \mathrm{m}$ response with high operation temperature. This novel MOS Ge/Si QDIP can increase the functionality of Si chip and is fully compatible with ULSI technology.

[1] C. W. Liu et al., IEDM Tech. Dig., pp.749-752, 1999.

[2] B.-C. Hsu et al., lEDM Tech. Dig., pp.91-94, 2002.

[3] Vinh Le Thanh el al., J. Vac. Sci. Technol. B 20(3), pp.1259-1265, 2002. 


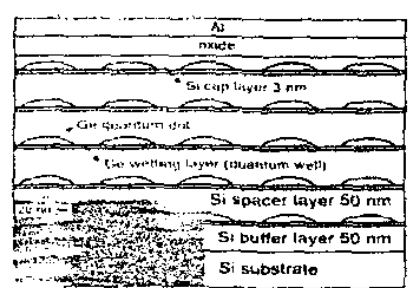

Fig. 1 The structure of MOS Ge/Si QDIP. The 5-layer $\mathrm{Ge}$ quantum dots were prepared by UHV/CVD.

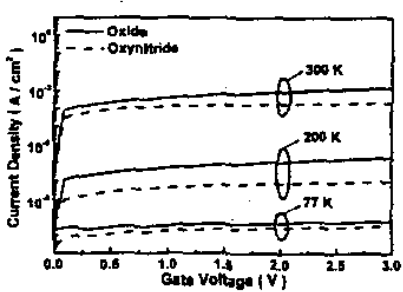

Fig. 4 The dark current of MOS Ge/S QDIPs with LPD oxide and oxynitride under different temperature. The device with oxynitride has lower dark curren density.

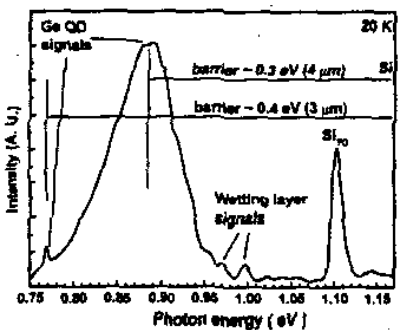

Fig. 7 The $20 \mathrm{~K}$ PL spectrum for a multilayer $\mathrm{Ge} / \mathrm{Si}$ quantum dot structure. Si bandgap is about $1.17 \mathrm{eV}$ at $20 \mathrm{~K}$. $\mathrm{QD}$ barrier is about $0.3 \sim 0.4 \mathrm{eV}$ $(4-3 \mu \mathrm{m})$.

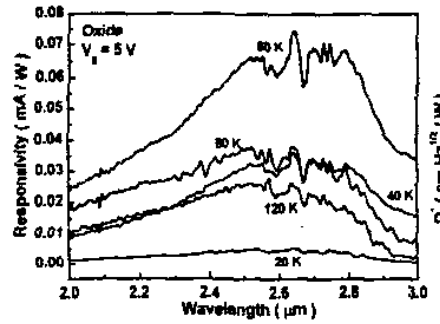

Fig. 10 Spectral responsivity at different temperature for $2-3 \mu \mathrm{m}$. The operating temperature reaches $120 \mathrm{~K}$.

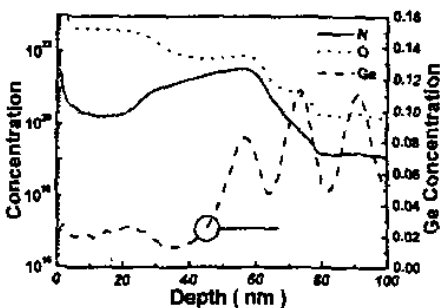

Fig, 2 The SIMS profile of as-deposited LPD-SiO $\mathrm{N}_{\mathrm{y}}$ film with thickness of $55 \mathrm{~nm}$. $\mathrm{O}: \mathrm{N}=16: 7$ at $\mathrm{N}$ peak position (SiON/Si interface).

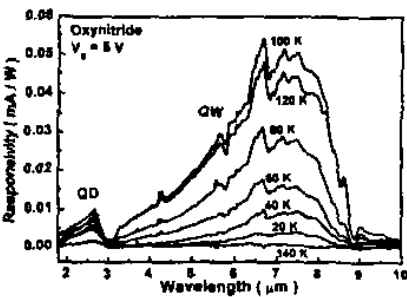

Fig. 5 Spectral responsivity of the device with oxynitride at different temperature. The operating temperature reaches $140 \mathrm{~K}$ for $3 \sim 10 \mu \mathrm{m}$ detection.

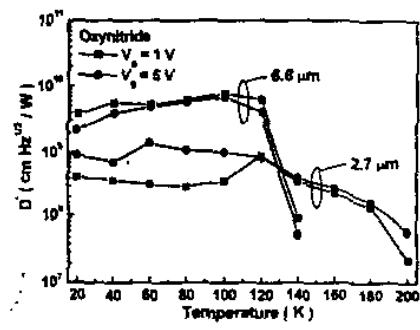

Fig. 8 The detectivity vs. temperature at $\mathrm{V}_{\mathrm{G}}=1 \mathrm{~V}$ and $\mathrm{V}_{\mathrm{G}}=5 \mathrm{~V}$ for QDiPs with oxynitride.

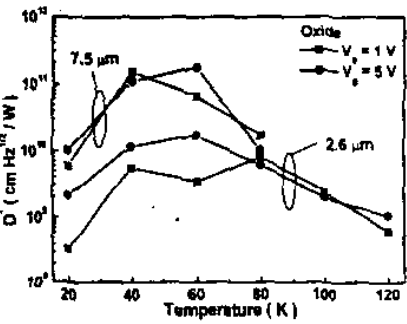

Fig. II The detectivity at $V_{G}=I V$ and $V_{G}=5 \mathrm{~V}$ for devices with oxide The peak detectivity is $2 \times 10^{11}$ $\mathrm{cm} \cdot \mathrm{Hz}^{1 / 2} / \mathrm{W}$.

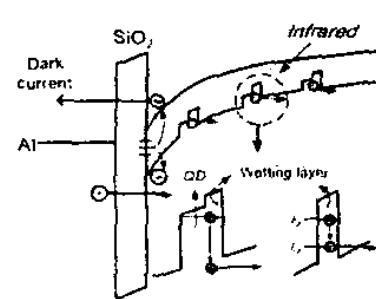

Fig. 3 The hand diagram of the device under inversion bias. The confined holes have transitions from $E_{1}-t o-E_{2}$ (yuantum well) and $E_{1}$-to-continuum (Q1) under infrared exposures.

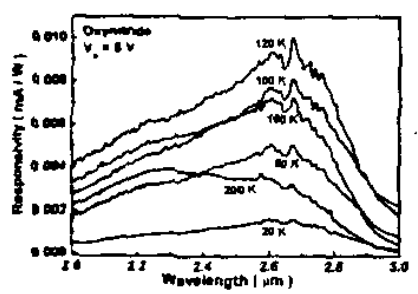

I If A Spectral responsivity of the disice with oxynitride at different ksmperiture for $2-3 \mu \mathrm{m}$. The operaling temperature is up to $200 \mathrm{~K}$.

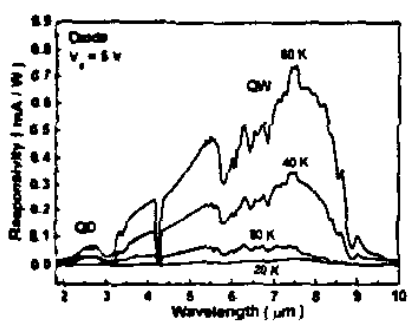

Fif 9 Spectral responsivity at ditterint ismprature. The operating iemprature of device with oxide reance, ko $\mathrm{A}$.

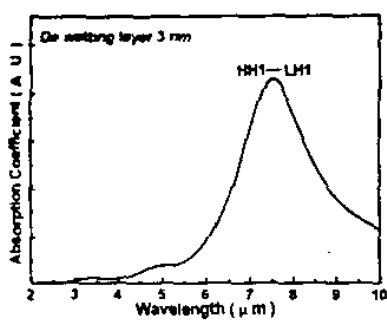

Fig. 12 The calculated absorption coeflicients of total intersubband transitions. A absorption peak is located at $7.5: 2 \mathrm{~m}$ due to the heavy hole to light hole transition. 